\title{
A Multimodal Gamified Platform for Real-Time User Feedback in Sports Performance
}

\author{
David Monaghana, Freddie Honohan a, Amin Ahmadia, \\ Troy McDaniel ${ }^{\mathrm{b}}$, Ramin Tadayon ${ }^{\mathrm{b}}$, Ajay Karpur ${ }^{\mathrm{b}}$, \\ Kieran Morana ${ }^{\mathrm{a}}$, Noel E. O'Connor ${ }^{\mathrm{a}}$ and Sethuraman Panchanathan ${ }^{\mathrm{b}}$ \\ a Insight Centre for Data Analytics, Dublin City University, Ireland \\ ${ }^{\mathrm{b}}$ Center for Cognitive Ubiquitous Computing, Arizona State University, Tempe, Arizona 85281 USA \\ monodavids@gmail.com
}

\begin{abstract}
In this paper we introduce a novel platform that utilises multi-modal low-cost motion capture technology for the delivery of real-time visual feedback for sports performance. This platform supports the expansion to multi-modal interfaces that utilise haptic and audio feedback, which scales effectively with motor task complexity. We demonstrate an implementation of our platform within the field of sports performance. The platform includes low-cost motion capture through a fusion technique, combining a Microsoft Kinect V2 with two wrist inertial sensors, which make use of the accelerometer and gyroscope sensors, alongside a game-based Graphical User Interface (GUI) for instruction, visual feedback and gamified score tracking.
\end{abstract}

\section{Categories and Subject Descriptors}

I.4.5 [Computing Methodologies]: Image Processing and Computer Vision-Reconstruction

\section{Keywords}

Motion capture, Human-Computer Interaction, Multimodal Interfaces, Sports Performance

\section{INTRODUCTION}

As sensing and feedback technology have become increasingly prevalent worldwide, motor learning has become a critical topic of study and research in the field of HumanComputer Interaction. Of particular interest is unsupervised motor learning, wherein motor function is improved in the absence of a trainer. To date, there remains a critical need in the rehabilitation applications for an effective automated

\footnotetext{
${ }^{*}$ This work was funded by the ASU-DCU Catalyst Fund. Part of this work was supported by Science Foundation Ireland under grant number SFI/12/RC/2289.
}

Permission to make digital or hard copies of part or all of this work for personal or classroom use is granted without fee provided that copies are not made or distributed for profit or commercial advantage and that copies bear this notice and the full citation on the first page. Copyrights for third-party components of this work must be honored. For all other uses, contact the owner/author(s).

MM'16 October 15-19, 2016, Amsterdam, Netherlands

(C) 2016 Copyright held by the owner/author(s).

ACM ISBN 978-1-4503-3603-1/16/10.

DOI: http://dx.doi.org/10.1145/2964284.2973815 method for motor learning in the home environment [12], or any such environment without the direct supervision of a qualified trainer. There are heavy limits on trainer availability in general, and consequently, unsupervised training is required outside of sports training sessions. Moreover, it is also apparent that guidance and frequent, informative feedback are necessary in real-time to catalyze motor learning [1]. Furthermore, motor tasks are often repetitive and monotonous, resulting in reduced compliance without some form of engagement or interaction [10].

Providing feedback on human motion presents a number of research challenges including: capturing bio-mechanically accurate motion outside a laboratory environment, automatic classification and extraction of different actions within a movement sequence, analysis and interpretation of motion data of each action, communication of the analysis results and suggestions for modification/improvement back to the end user. The feedback that is delivered to the end user also depends upon a number of factors including the nature of the movement being measured (e.g. day-long activity vs a specific exercise), complexity of the action (e.g. simple single limb movement vs whole body action), user context (e.g. location and time) and user experience (e.g. novice vs expert). A further over-arching research challenge of course is that all of this should be accomplished in real-time (i.e. during a movement) or near real-time (i.e. immediately after a movement has been performed).

In this paper, we present the design of an interactive platform for motion sensing and feedback delivery in a home based setting, which is capable of delivering real-time visual feedback, with an emphasis on performance.

\section{MOTION CAPTURE}

Motion Capture (MoCap) is a field of science that primarily deals with the recording, reconstructing and analysis of motion, and is a well-studied and broad area of research [13]. Marker based Vicon MoCap ${ }^{1}$ generally represents the gold-standard and offers excellent results but carries a high price tag and requires complex post-processing, leaving it out of reach for most. For this reason, significant research is being performed into the area of low-cost alternatives [8, 6], using either marker-less based methods such computer vision [14] based analysis or by using depth cameras like the Microsoft Kinect [7, 15]. However, many of these systems suffer from tracking errors due to marker occlusions.

\footnotetext{
$\overline{{ }^{1} \text { See: http://www.vicon.com/ }}$
} 


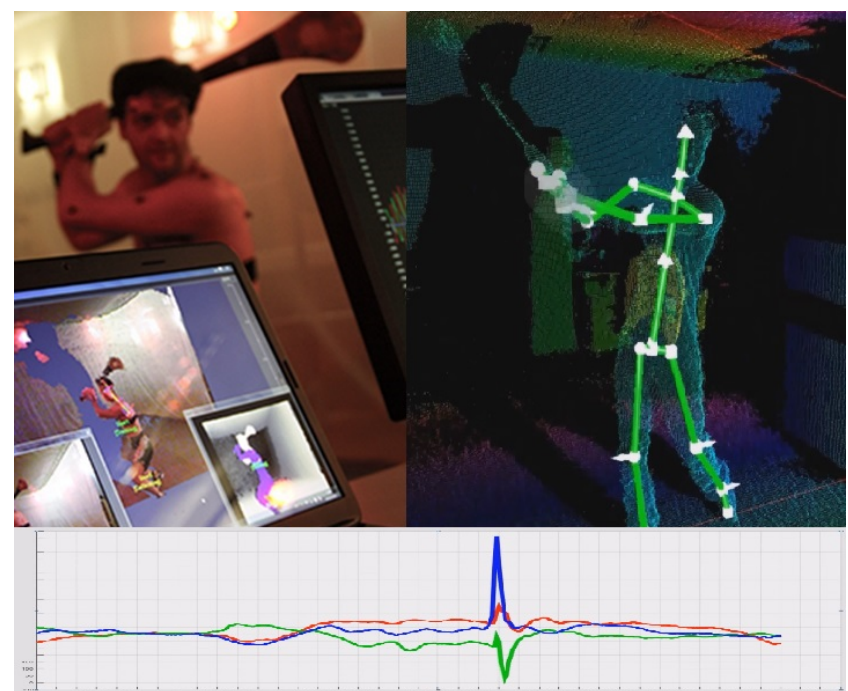

Figure 1: The authors demonstrating the comparison between a Gold-Standard Vicon system, on the left, with their low cost Kinect-IMU fusion approach.

Inertial Measurement Units (IMUs) offer an accurate MoCap alternative $[9,3]$ and have been developed into commercially successful systems, such as XSens [11], however they are still prone to drift in accuracy over time, a common limitation of inertial sensing. IMUs are low power, light weight, offer high sample rates and do not suffer from occlusions, but they are susceptible to orientation and position errors if not corrected over time. Furthermore, commercially available IMU MoCap solutions are still relatively high in price, approx. 50K Euro. During the last few years, a number of low-cost alternatives have been proposed, the majority of which are based upon fusing data received from different cheap sensor modalities (e.g. fusion of Kinect \& wearables) $[5,4]$ to provide inexpensive yet accurate systems.

Previous research [2] carried out by the authors has investigated a low-cost platform that utilises 3 IMU sensors together with advanced analysis algorithms, multiple IMU calibration and inverse kinematics for detecting the stance phase of the gait cycle. This allows for the reduction of integration drift error of the IMU system and creates an accurate 3-D gait analysis platform for the lower limbs.

In this paper the described platform utilises low-cost sensor fusion to produce a skeletal reconstruction that combines the global joint positioning of the Microsoft Kinect together with the joint-rotational accuracy of inertial sensors.

\section{THE PLATFORM}

The platform consists of two IMUs, a Microsoft Kinect V2 and software written in C\# through the open-source Unity3D game engine. The IMUs are connected via Bluetooth to a computer and are set with a sample frequency of $256 \mathrm{~Hz}$. Only the accelerometer and gyroscope channels are used. After a brief T-pose to calibrate the IMUs to the local coordinate system of the Kinect, a skeleton fusion technique is employed, based on $[2,5,4]$, that uses the skeleton extraction/tracking from the Microsoft Kinect for all joints except the wrists where the joint position and orientations are calculated from the IMUs (note that the Hand, HandTip and Thumb joints from the Kinect SDK were not used here). The platform can be seen in Figure 2.

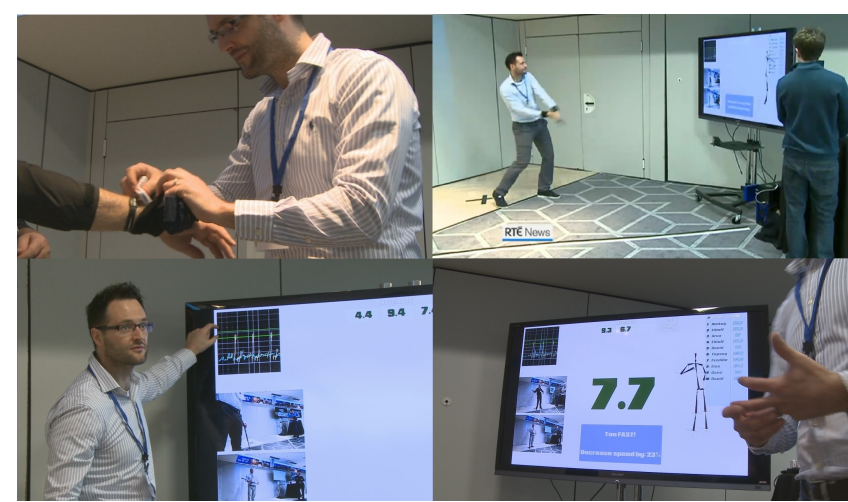

Figure 2: Clockwise from the top left: An IMU is strapped to each wrist of the user's wrists for use with the platform; the user is being recorded using the platform whilst attempting to copy the selected motion; Accelerometer data graphed against the target acceleration of the correct technique is displayed for the user; A gamified score feedback is given to the user after each of their three attempts and a leader-board of the top scores records the total.

A sample movement of a trainer or athlete performing a desired motion is demonstrated for the user through a video, skeleton visualisation and an IMU acceleration graph. The user is then prompted to perform the movement. A comparison module compares the user's accelerations from each wrist to the sample movement and visual feedback is given. A score based on the a ratio of the user data to the sample data is recorded for this attempt and feedback is given instructing the user to speed up or slow their movement. The user performs two subsequent attempts and then the overall score is recorded on a system score board for comparison. This platform demonstrates an example of providing feedback soon after a movement, where the goal is not to change the technique as it is being produced but rather to indicate to an end user that he/she has attained a specific performance level associated with a fast complex movement.

\section{CONCLUSION}

We wish to understand the most optimum way of delivering multimodal feedback to a user both during slow moving rehabilitation sessions and also throughout fast moving sporting sessions. If a user can be informed through out a session about their performance, this can allow them to make the necessary adjustments to their technique in order to perform better, both in sports performance and in muscular-skeletal rehabilitation.

\section{DEMONSTRATION}

The demonstration will consist of a laptop, a Kinect, two wrist worn IMUs, a bluetooth receiver and a large screen. The platform will be showcased using a pre-recorded sporting movement from an Irish national Hurling champion and users will be shown how to engage with the system in order to replicate the precise sporting motion. Then they can proceed to play the game, having three attempts and finally being given a score based on their performance. A poster detailing the motivations behind and current state of our research on low-cost motion capture and gaming technologies will also be shown. 


\section{REFERENCES}

[1] Patient Motivation and Adherence to Postsurgery Rehabilitation Exercise Recommendations: The Influence of Physiotherapists' Autonomy-Supportive Behaviors. 90.

[2] A. Ahmadi, F. Destelle, D. Monaghan, K. Moran, N. E. O'Connor, L. Unzueta, and M. Linaza. Human gait monitoring using body-worn inertial sensors and kinematic modelling. pages 1-4, 2015.

[3] A. Ahmadi, E. Mitchell, C. Richter, F. Destelle, M. Gowing, N. E. O'Connor, and K. Moran. Toward automatic activity classification and movement assessment during a sports training session. Internet of Things Journal, IEEE, 2(1):23-32, 2015.

[4] F. Destelle, A. Ahmadi, K. Moran, N. E. O'Connor, N. Zioulis, A. Chatzitofis, D. Zarpalas, P. Daras, L. Unzueta, and J. Goenetxea. A multi-modal 3d capturing platform for learning and preservation of traditional sports and games. ACM, pages $747-748$, 2015.

[5] F. Destelle, A. Ahmadi, N. E. O'Connor, K. Moran, A. Chatzitofis, D. Zarpalas, and P. Daras. Low-cost accurate skeleton tracking based on fusion of kinect and wearable inertial sensors. pages 371-375, 2014.

[6] J. E. Deutsch, M. Borbely, J. Filler, K. Huhn, and P. Guarrera-Bowlby. Use of a low-cost, commercially available gaming console (wii) for rehabilitation of an adolescent with cerebral palsy. Physical Therapy, 88:1196-1207, 2008.

[7] S. Essid, D. Alexiadis, R. Tournemenne, M. Gowing, P. Kelly, D. Monaghan, P. Daras, A. Drémeau, and N. E. O'Connor. An advanced virtual dance performance evaluator. IEEE, 2012.
[8] E. Farella, L. Benini, B. Ricco, and A. Acquaviva. A low-power, low-cost motion capture system based on integrated accelerometers. Advances in Multimedia, 1, 2007.

[9] H. Liu, X. Wei, J. Chai, I. Ha, and T. Rhee. Realtime human motion control with a small number of inertial sensors. Symposium on Interactive 3D Graphics and Games. ACM, pages 133-140, 2011.

[10] D. Reinkensmeyer and S. Housman. "If I can't do it once, why do it a hundred times?": Connecting volition to movement success in a virtual environment motivates people to exercise the arm after stroke. In Virtual Rehabilitation, 2007, pages 44-48, Sept. 2007.

[11] D. Roetenberg, H. Luinge, and P. Slycke. Xsens mvn: Full 6dof human motion tracking using miniature inertial sensors. Xsens Motion Technologies BV, Tech.Rep., 2009.

[12] N. SkjÃęret, A. Nawaz, T. Morat, D. Schoene, J. L. Helbostad, and B. Vereijken. Exercise and rehabilitation delivered through exergames in older adults: An integrative review of technologies, safety and efficacy. International Journal of Medical Informatics, 85(1):1-16, Jan. 2016.

[13] G. Welch and E. Foxlin. Motion tracking: No silver bullet, but a respectable arsenal. Computer Graphics and Applications, IEEE, 22:24-38, 2002.

[14] M. Windolf, N. GÃützen, and M. Morlock. Systematic accuracy and precision analysis of video motion capturing systemsâĂTexemplified on the vicon-460 system. Journal of Biomechanics, 41:2776-2780, 2008.

[15] Z. Zhang. Microsoft kinect sensor and its effect. MultiMedia, IEEE, 19:4-10, 2012. 